\section{Suppressive effects of anagrelide on cell cycle progression and the maturation of megakaryocyte progenitor cell lines in human induced pluripotent stem cells}

Anagrelide is widely used as a therapeutic agent for patients with essential thrombocythemia (ET). ${ }^{1}$ It was initially developed as an antiplatelet aggregation drug by inhibition of phosphodiesterase III, ${ }^{2}$ and its primary effect at therapeutic concentrations is on the post-mitotic phase of megakaryocyte development, where it reduces platelet production by reducing the size and ploidy of megakaryocytes. This then induces a thrombocytopenic effect ${ }^{3,4}$ in patients with thrombocythemia; however, the plateletlowering effect of anagrelide is not well understood. In a clinical study that used bone marrow samples obtained from patients with ET, anagrelide therapy reduced the number of circulating platelets by reducing both the hyperproliferation and the differentiation of megakaryocytes.

Megakaryocytic cell lines are usually premature cells that cannot transform into mature megakaryocytes and generate platelets. Because of this, some conventional studies of megakaryocytes and platelets using cell lines or primary cells have proved to be problematic. In the present study, we used immortalized megakaryocyte progenitor cell lines (imMKCL), which were recently established from human induced pluripotent stem (iPS) cells, to elucidate the molecular mechanism of anagrelide in inhibiting platelet production.

We prepared the imMKCL as follows. Doxycyclineinducible lentiviral vectors harboring $c-M Y C, B M I 1$, and $B C L-X L$ were introduced into the imMKCL to clinically produce artificially generated platelets. ${ }^{6-8}$ After removing the doxycycline, three over-expressed transgenes were turned off; the cells began to differentiate, and the platelets were generated in approximately 5-7 days (Figure 1A). To enhance the platelet production, the following compounds were added on day 0: an aryl hydrocarbon receptor antagonist (SR1; Merck Millipore, MA, USA), a ROCK inhibitor (Y-27632; Wako, Tokyo, Japan), and KP-457 (Kaken Pharmaceutical Co. Ltd., Tokyo, Japan) under a Material Transfer Agreement. KP-457 efficiently enables the retention of glycoprotein $\mathrm{Ib}$ (GPIb), also known as CD42. Without it, the loss of the GPIb extracellular domain would attenuate the ability of platelets to adhere to the extracellular matrix and form thrombi. ${ }^{9}$

The first experiment was to examine the proliferation of imMKCL and the inhibition of platelet production by treatment with anagrelide. Treating imMKCL with anagrelide for two days resulted in no remarkable morphological changes in cells in the undifferentiated (nonplatelet-producing) state; however, in cells in the differentiated (platelet-producing) state, those treated with anagrelide appeared to be mononuclear, whereas the untreated cells did not. This trend became clearer at day 6 (Figure 1B). MTS (3-(4,5-dimethylthiazol-2-yl)-5-(3-carboxymethoxyphenyl)-2-(4-sulfophenyl)-2H-tetrazolium, inner salt) assays were performed to test the effects of anagrelide treatment on the proliferation of imMKCL and non-megakaryocytic lineage cell lines (HL-60, THP-1, and K562). With anagrelide treatment, the cell growth curve for imMKCL in the undifferentiated state showed a significant decrease in absorbance at 96 hours (anagrelide 0 vs. $1 \mu \mathrm{M}, P<0.001$; anagrelide 0 vs. $10 \mu \mathrm{M}, P<0.01)$ (Figure 1C). Conversely, anagrelide treatment did not affect the proliferation of the non-megakaryocytic line- age cell lines (Online Supplementary Figure S1). In the differentiated state, proplatelets generated from imMKCL affected the absorbance in the MTS assay (data not shown); therefore, the cell proliferation of imMKCL in the differentiated state was evaluated using a bromodeoxyuridine $(\mathrm{BrdU})$ incorporation assay. This showed a significant decrease in the incorporation rate with anagrelide treatment (anagrelide 0 vs. 1 or $10 \mu \mathrm{M}$, both $P<0.001$ ) (Figure 1D), which suggested that anagrelide suppressed DNA synthesis during differentiation. To confirm the suppressive effects of anagrelide treatment on the maturation and proliferation of imMKCL, we investigated changes in mRNA expression of the megakaryocytic surface markers ITGA2B (CD41) and ITGB3 (CD61). This showed significant decreases in their expression in both the undifferentiated state $(P<0.05$ and $P<0.001$, respectively) and differentiated state $(P<0.01$ and $P<0.001$, respectively) (Figure $1 \mathrm{E})$. The resultant generation of mature platelets from imMKCL cultured for seven days in platelet-producing conditions was analyzed using flow cytometry. Mature platelets were detected as double-positive plots for CD41 and CD42b, the representative markers of mature and functional platelets. ${ }^{7}$ When $1 \mu \mathrm{M}$ of anagrelide was added to the culture on day 0 , this resulted in a significant decrease in the relative number of mature platelets $(P<0.001)$ (Figure $1 \mathrm{~F})$.

We went on to elucidate the molecular mechanism underlying anagrelide's platelet-lowering effect. To do this, RNA sequencing was conducted for imMKCL in both undifferentiated and differentiated states, comparing the cells with or without anagrelide treatment. The genes that were up-regulated by more than 2 -fold or down-regulated to less than half compared to control were identified and divided into six groups (Figure 2A). All the annotated terms in a gene ontology analysis that showed statistical significance $(P<0.05)$ were categorized as up-regulated and down-regulated genes (Figure $2 \mathrm{~B}$ and $\mathrm{C}$, respectively). In both states, the up-regulated genes showed a response to cyclic 3',5'-adenosine monophosphate (cAMP) consistent with the previously described mechanism of anagrelide action. ${ }^{2}$ Of note, the genes related to negative apoptotic process regulation were upregulated only in the differentiated state, which suggested that anagrelide affects megakaryocytic cells differently depending on their differentiation stages. The gene ontology analysis also demonstrated that gene sets related to platelet activation and degranulation were significantly down-regulated in both undifferentiated and differentiated states. In addition, gene set enrichment analysis showed that anagrelide treatment suppressed gene sets related to the cell cycle and maturation, such as mitosis and DNA replication, as well as platelet-related genes (Figure 3A and $\mathrm{B}$ ). The expressions of some megakaryocyte-related genes were evaluated using realtime quantitative polymerase chain reaction. TRIB3 (tribbles homolog 3) expression, which has been reported to be a negative modulator of megakaryopoiesis and platelet generation, ${ }^{10}$ was significantly reduced with differentiation of imMKCL $(P<0.01)$; its expression was increased in the cells receiving anagrelide treatment relative to the cells that did not $(P<0.05)$. The expression of PF4 (platelet factor 4), a chemokine found in alpha granules and released from activated platelets during aggregation related to wound healing and inflammation, ${ }^{11}$ was markedly increased with differentiation of imMKCL $(P<0.001)$; its expression was reduced in the cells receiving anagrelide treatment relative to the cells that did not in both undifferentiated and differentiated states $(P<0.05$ and $P<0.001$, respectively) (Figure 3C). 
A

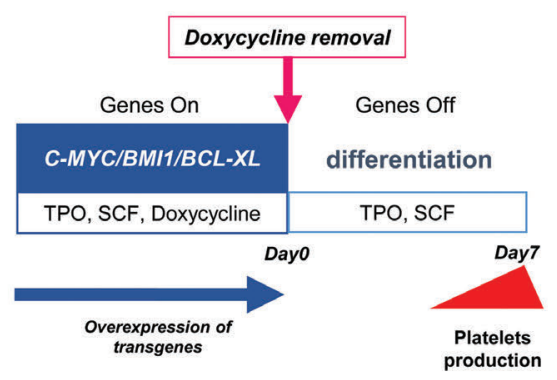

B

\begin{tabular}{|c|c|c|c|}
\hline \multirow[b]{2}{*}{ Anagrelide } & $\begin{array}{l}\text { Undifferentiated } \\
\text { (Non platelets } \\
\text { producing) }\end{array}$ & \multicolumn{2}{|c|}{$\begin{array}{c}\text { Differentiated } \\
\text { (Platelets producing) }\end{array}$} \\
\hline & Day2 & Day2 & Day6 \\
\hline \multicolumn{4}{|l|}{$0 \mu \mathrm{M}$} \\
\hline \multirow[t]{2}{*}{$1 \mu \mathrm{M}$} & & & \\
\hline & & & 28 \\
\hline
\end{tabular}

C

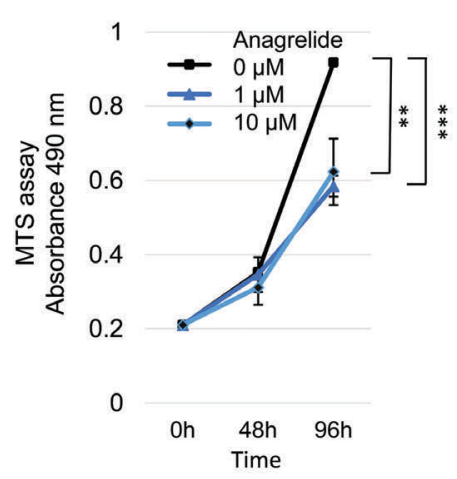

D

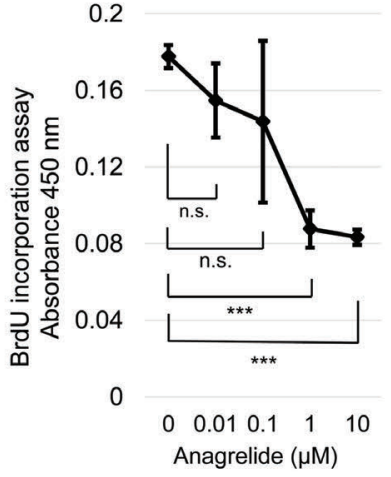

E

ITGA2B

ITGB3

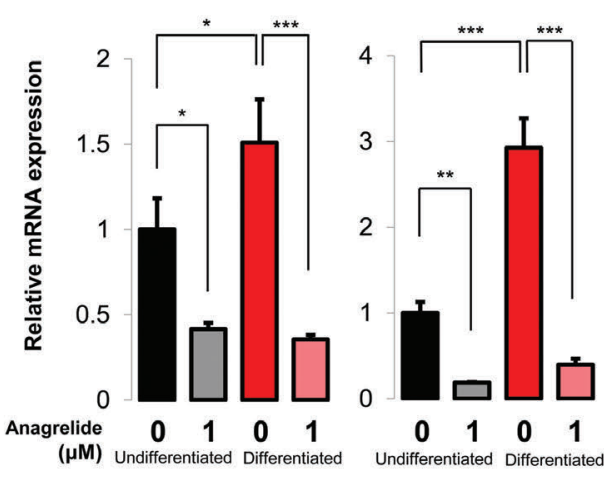

F

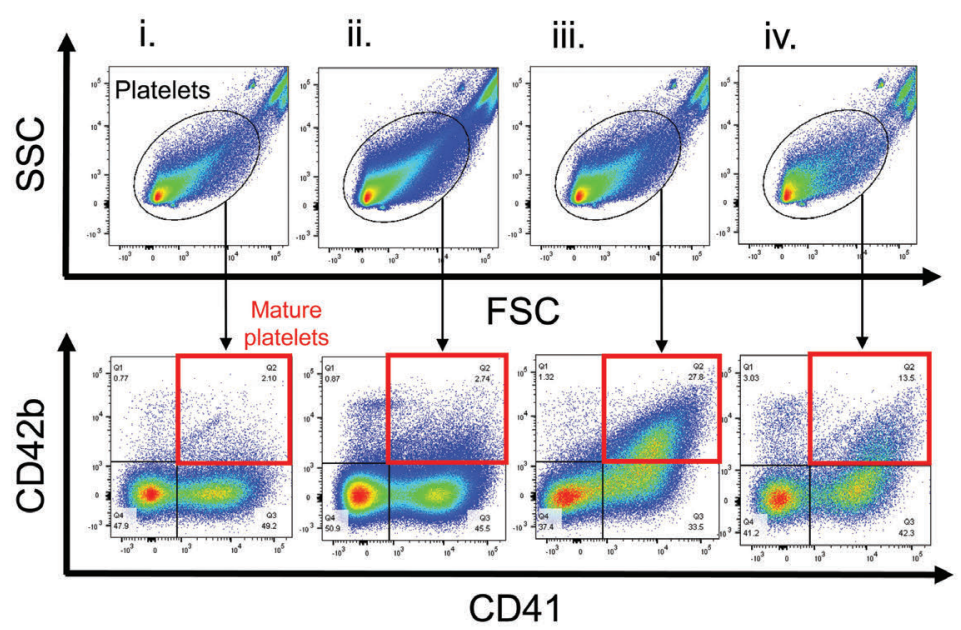

\begin{tabular}{l|cccc} 
& i & ii & ii & iv \\
\hline Doxycycline & + & - & - & - \\
\hline KP457 & - & - & + & + \\
\hline Anagrelide $(\mu \mathrm{M})$ & 0 & 0 & 0 & 1
\end{tabular}

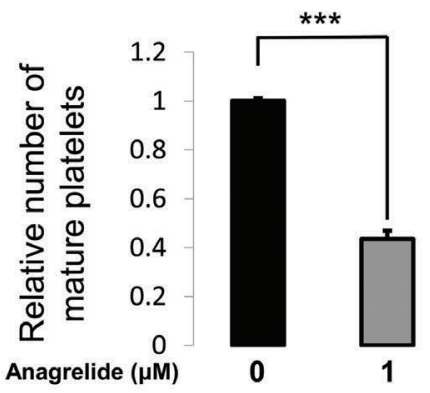

Figure 1. Suppressive effects of anagrelide treatment on immortalized megakaryocyte progenitor cell lines (imMKCL). (A) Schema for platelet production by imMKCL. (B) Morphological change in imMKCL is shown in several conditions. The cells were stained with Wright-Giemsa staining. (C) imMKCL treated with various concentrations of anagrelide were analyzed using an MTS (3-(4,5-dimethylthiazol-2-yl)-5-(3-carboxymethoxyphenyl)-2-(4-sulfophenyl)-2H-tetrazolium, inner salt) assay at 0,48 , and 96 hours (h) in the undifferentiated state. Data are expressed as the mean \pm standard deviation (SD) from three independent experiments ( $* * P<0.01 ; * * * P<0.001$; one-way ANOVA with post-hoc Bonferroni test). (D) imMKCL treated with anagrelide were analyzed using a bromodeoxyuridine (BrdU) incorporation assay at $48 \mathrm{~h}$ in the differentiated state. Data are expressed as mean $\pm \mathrm{SD}$ from three independent experiments ( $* * * P<0.001$; one-way ANOVA with post-hoc Bonferroni test). (E) mRNA expressions of ITGA2B (CD41) and ITGB3 (CD61) in imMKCL treated with 0 or $1 \mu \mathrm{M}$ of anagrelide for two days were analyzed using quantitative real-time polymerase chain reaction. Data are expressed as mean $\pm \mathrm{SD}$ from three independent experiments $(* P<0.05$; $* * P<0.01 ; * * * P<0.001$; one-way ANOVA with post-hoc Bonferroni test). (F) The platelets generated by imMKCL were analyzed by flow cytometry seven days after removing doxycycline from the culture. The generated platelets are plotted in ellipses in the upper four panels, and expanded in the lower four panels according to CD41 and CD42b positivity. The four different culture conditions are shown in the small table top right. The bar graph shows the changes in the relative number of mature platelets of (iii) and (iv). Data are expressed as mean \pm SD from three independent experiments ( $* * * P<0.001$; Student $t$-test). ns: not significant. 
A cell cycle analysis was performed to confirm the suppressive changes in cell cycle-related genes resulting from anagrelide treatment. The number of imMKCL in the $S$ phase decreased in the undifferentiated state (Figure 3D), whereas fewer cells were found in the differentiated state with or without anagrelide treatment (Online Supplementary Figure S2). Few cells were detected as

A

\section{Upregulated genes}

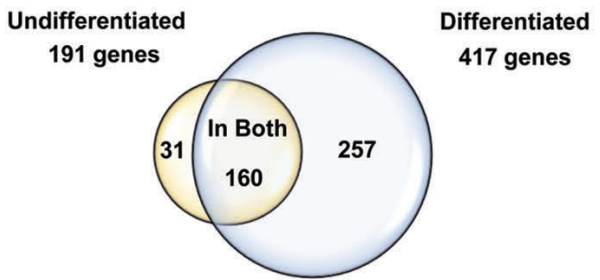

B

$$
\text { Upregulated genes }
$$

Only in Undifferentiated (31 genes)

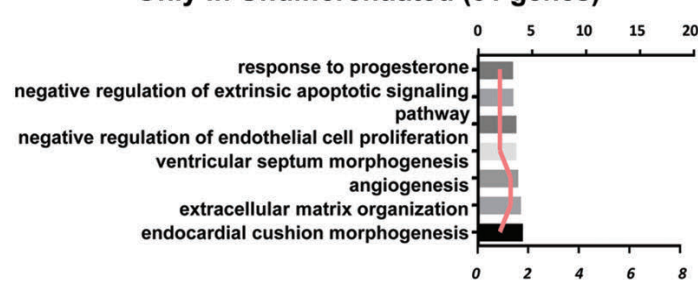

Only in Differentiated (257 genes)

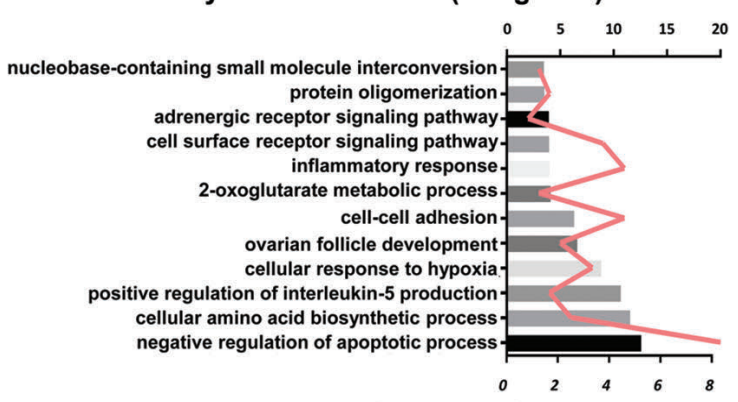

In Both (160 genes)

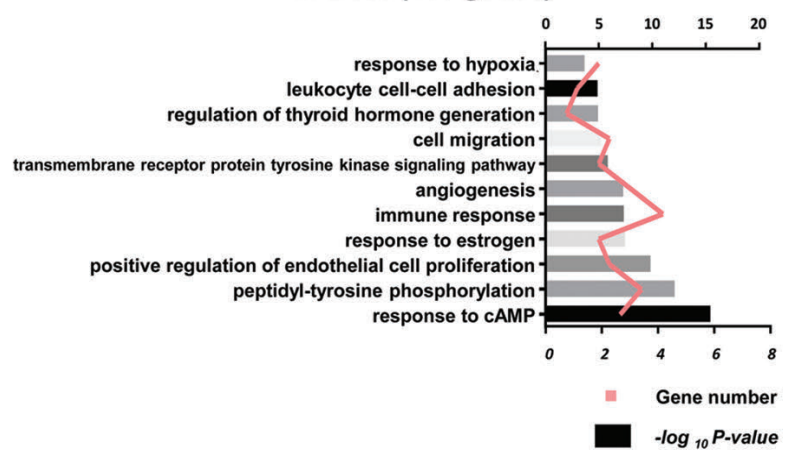

dead; i.e. even with anagrelide treatment at a relatively high concentration $(10 \mu \mathrm{M})$, few cells underwent apoptosis in both undifferentiated and differentiated states. This result was confirmed with an apoptosis assay, which showed fewer cells in the early or late apoptotic period in both undifferentiated and differentiated states (Online Supplementary Figure S3).

\section{Downregulated genes}

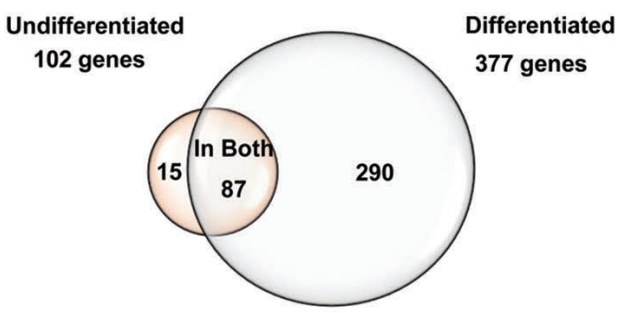

C

\section{Downregulated genes}

Only in Undifferentiated (15 genes)

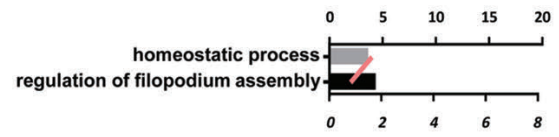

Only in Differentiated (290 genes)

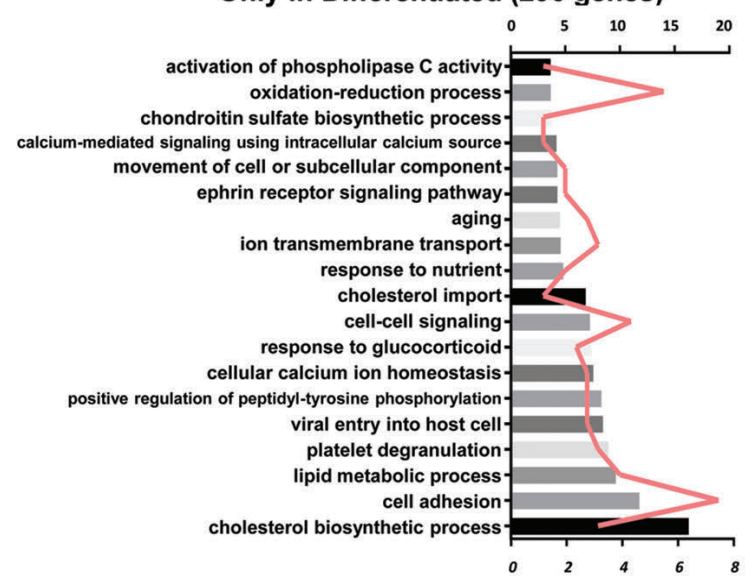

In Both (87 genes)

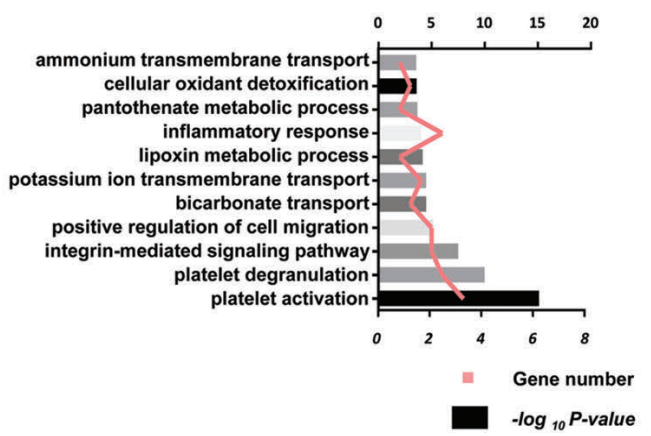

Figure 2. RNA sequencing of immortalized megakaryocyte progenitor cell lines (imMKCL) treated with anagrelide and the subsequent gene ontology analysis. (A) RNA sequencing was conducted for imMKCL treated for two days with $1 \mu \mathrm{M}$ anagrelide or without the drug (control). The resulting Venn diagrams show overlaps of genes up-regulated by more than two-fold and down-regulated to less than one-half compared to control. (B) The result of the gene ontology analysis of up-regulated genes is shown in the three groups: in the undifferentiated state, in the differentiated state, and in both states. The pink lines show the number of genes (upper scale) and grayscale bars show $-\log _{10} P$ values (bottom scale). (C) The results of the gene ontology analysis of down-regulated genes were the same as for the up-regulated genes. 
In a previous study of cell cycle-related genes, Apostolidis et al. ${ }^{12}$ showed that the upregulation of TP53 (a well-known cell cycle-regulating gene coding p53) and downstream genes such as CDKN1A (coding p21), and the subsequent suppression of CCND3 coding cyclin D3, resulted in cell arrest in the G1 phase in megakaryocytic cells. They also demonstrated that the loss of p53 result- ed in increased polyploidization during megakaryopoiesis..$^{13}$ During an attempt to accelerate megakaryocyte differentiation, Zou et al. ${ }^{14}$ found that suppressive changes in $\mathrm{p} 53$, and the subsequent suppression of $\mathrm{p} 21$, induced megakaryocyte proliferation and maturation. These findings indicate that the upregulation of p53 and subsequent changes in downstream genes are associated
A

\begin{tabular}{|c|c|c|}
\hline \multirow{2}{*}{ Downregulated } & \multicolumn{2}{|c|}{$\begin{array}{c}\text { NES } \\
\text { NOM p-value/FDR q-value }\end{array}$} \\
\hline & Undifferentiated & Differentiated \\
\hline Chang_Cycling_Genes & $\begin{array}{c}-2.24 \\
0.000 / 0.000\end{array}$ & $\begin{array}{c}-1.58 \\
0.002 / 0.160\end{array}$ \\
\hline Reactome_DNA_Replication & $\begin{array}{c}-2.09 \\
0.000 / 0.002\end{array}$ & $\begin{array}{c}-1.56 \\
0.008 / 0.171\end{array}$ \\
\hline Reactome_Mitotic_M_M_G1_Phases & $\begin{array}{c}-2.07 \\
0.000 / 0.002\end{array}$ & $\begin{array}{c}-1.62 \\
0.002 / 0.159\end{array}$ \\
\hline Reichert_Mitosis_LIN9_Targets & $\begin{array}{c}-2.07 \\
0.000 / 0.002\end{array}$ & $\begin{array}{c}-1.74 \\
0.004 / 0.109\end{array}$ \\
\hline Kong_E2F3_Targets & $\begin{array}{c}-2.02 \\
0.000 / 0.003\end{array}$ & $\begin{array}{c}-1.58 \\
0.006 / 0.162\end{array}$ \\
\hline Reactome_Platelet_Homeostasis & $\begin{array}{c}-1.98 \\
0.000 / 0.004\end{array}$ & $\begin{array}{c}-1.33 \\
0.090 / 0.356\end{array}$ \\
\hline Reactome_Mitotic_Prometaphase & $\begin{array}{c}-1.93 \\
0.000 / 0.007\end{array}$ & $\begin{array}{c}-1.60 \\
0.004 / 0.164\end{array}$ \\
\hline Molenaar_Targets_of_CCND1_and_CDK4_DN & $\begin{array}{c}-1.90 \\
0.000 / 0.007\end{array}$ & $\begin{array}{c}-1.61 \\
0.010 / 0.161\end{array}$ \\
\hline Reactome_Cell_Cycle_Mitotic & $\begin{array}{c}-1.90 \\
0.000 / 0.010\end{array}$ & $\begin{array}{c}-1.22 \\
0.083 / 0.439\end{array}$ \\
\hline Raghavachari_Platelet_Spesific_Genes & $\begin{array}{c}-1.61 \\
0.014 / 0.130\end{array}$ & $\begin{array}{c}-1.95 \\
0.000 / 0.065\end{array}$ \\
\hline Senese_HDAC2_Targets_DN & $\begin{array}{c}-1.44 \\
0.028 / 0.273\end{array}$ & $\begin{array}{c}-1.88 \\
0.000 / 0.040\end{array}$ \\
\hline Reactome_Chromosome_Maintenance & $\begin{array}{c}-1.40 \\
0.007 / 0.310\end{array}$ & $\begin{array}{c}-1.74 \\
0.000 / 0.105\end{array}$ \\
\hline
\end{tabular}

B

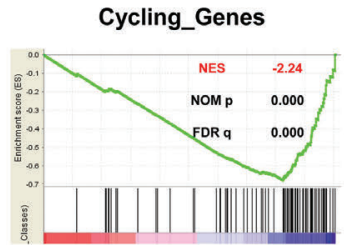

DNA_Replication

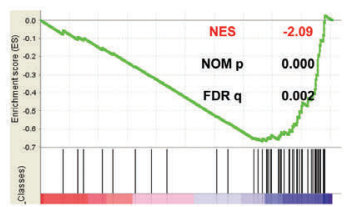

Mitotic_M_M_G1_Phases

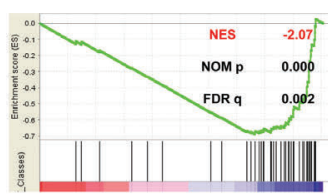

Mitosis_LIN9_Targets

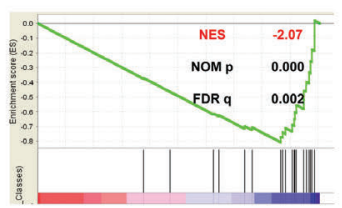

E2F3_Targets

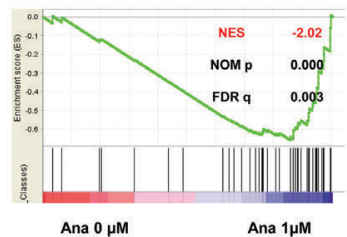

- Enrichment profile - Hits
C

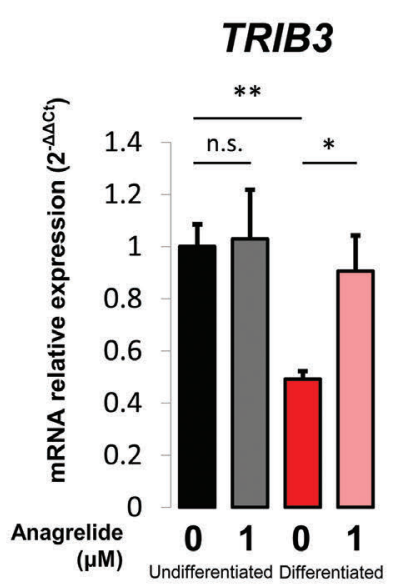

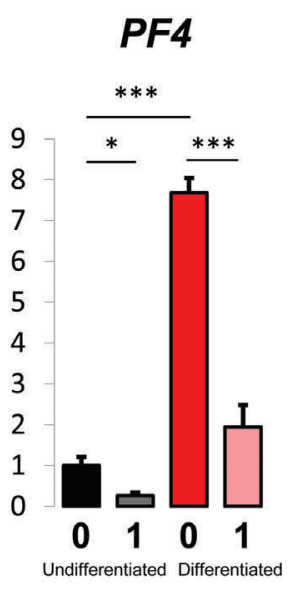

D

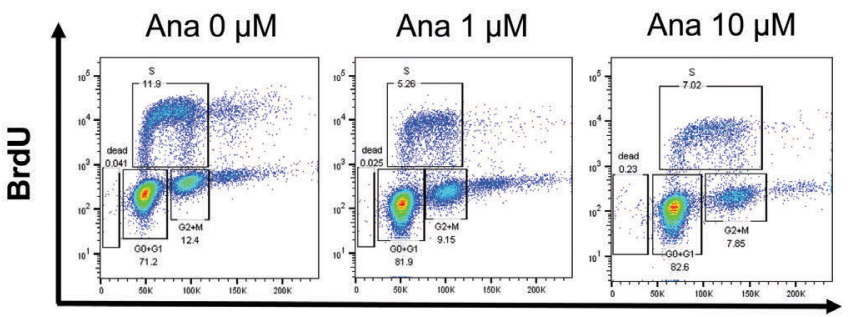

7AAD

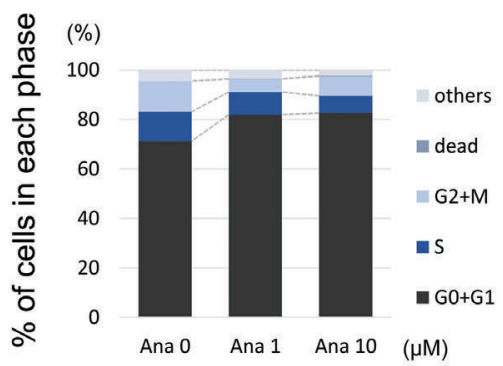

Figure 3. Gene set enrichment analysis (GSEA) and cell cycle analysis. (A) GSEA for the down-regulated genes in immortalized megakaryocyte progenitor cell lines (imMKCL) in the undifferentiated and differentiated states, according to anagrelide treatment. Normalized enrichment scores, nominal (NOM) P-values, and false discovery rate q-values are indicated for each cell. Deeper blue represents stronger downregulation of the gene sets. (B) GSEA plots of the representative down-regulated gene sets. (C) The results of quantitative quantitative real-time polymerase chain reaction for the mRNA expression of the megakaryocyterelated genes TRIB3 and PF4 in imMKCL treated with 0 or $1 \mu \mathrm{M}$ anagrelide for two days. Data are expressed as the mean \pm standard deviation (SD) from three independent experiments ( $* P<0.05 ; * * P<0.01 ; * * * P<0.001$, by one-way ANOVA with post-hoc Bonferroni test). (D) Results of cell cycle analysis of imMKCL treated with 0, 1 or $10 \mu \mathrm{M}$ anagrelide for two days. The analysis was performed by labeling with 7AAD stained for DNA content and bromodeoxyuridine (BrdU) stained for cells in the $\mathrm{S}$ phase. The scatter plot histograms show 7AAD (x-axis) and BrdU (y-axis) in imMKCL in the undifferentiated state. Bottom bar graph shows the percentage of cells in each phase. ns: not significant. 
with the suppressive effect on megakaryocytes. It has also been shown using cord blood-derived $\mathrm{CD}_{3} 4^{+}$cells that upregulation of the eIF $2 \alpha /$ ATF pathway is an important underlying mechanism of anagrelide's action, suppressing the growth of megakaryocytic cells. ${ }^{15}$ It has also been demonstrated that silencing TRIB3 facilitated megakaryocyte differentiation and, conversely, that TRIB3 overexpression inhibited the differentiation process in the primary hematopoietic cell culture. ${ }^{10} \mathrm{We}$ hope that further research using our novel cell lines, imMKCL, will strengthen these previous findings and also clarify more specific mechanisms of anagrelide.

In summary, the platelet-lowering effect of anagrelide was successfully reproduced using imMKCL, established from human iPS cells, that generated functional platelets. The findings revealed that anagrelide specifically suppressed genes associated with megakaryopoiesis and platelet formation. These suppressive effects were caused by regulation of the cell cycle, not through the apoptotic process. The results also demonstrated that imMKCL have the advantage of being able to withstand analyses performed separately in the undifferentiated and differentiated stages of megakaryocytic cells and to generate functional platelets, indicating their value as a model for human megakaryopoiesis and platelet formation.

Koji Takaishi, ${ }^{1}$ Masahiro Takeuchi, ${ }^{1}$ Shokichi Tsukamoto, Naoya Takayama, Motohiko Oshima, Kenji Kimura, Yusuke Isshiki, Kensuke Kayamori, ${ }^{1}$ Yutaro Hino, Nagisa Oshima-Hasegawa, ${ }^{1}$ Shio Mitsukawa, ${ }^{1,4}$ Yusuke Takeda, ${ }^{1}$ Naoya Mimura, ${ }^{1,4}$ Chikako Ohwada, Tohru Iseki, 1,4 Sou Nakamura, Koji Eto, 2,5 Atsushi Iwama, ${ }^{3}$ Koutaro Yokote, ${ }^{6}$ Chiaki Nakaseko ${ }^{1,7}$ and Emiko Sakaida ${ }^{1}$

'Department of Hematology, Chiba University Hospital, Chiba; ${ }^{2}$ Department of Regenerative Medicine, Chiba University Graduate School of Medicine, Chiba; ${ }^{3}$ Division of Stem Cell and Molecular Medicine, Center for Stem Cell Biology and Regenerative Medicine, Institute of Medical Science, University of Tokyo, Tokyo; ${ }^{4}$ Department of Transfusion Medicine and Cell Therapy, Chiba University Hospital, Chiba; Department of Clinical Application, Center for iPS Cell Research and Application (CiRA), Kyoto University, Kyoto; ${ }^{6}$ Department of Clinical Biology and Medicine, Chiba University Graduate School of Medicine, Chiba and 'Department of Hematology, International University of Health and Welfare School of Medicine, Narita, Japan

Acknowledgments: the authors would like to thank JSPS KAKENHI Grant in Aid for Scientific Research (C) (\#17K09901) and MARUZEN-YUSHODO Co. Ltd. for English Language editing (http://kw.maruzen.co.jp/kousei-honyaku/).

Correspondence: EMIKO SAKAIDA

esakaida@faculty.chiba-u.jp

doi:10.3324/haematol.2018.214841
Information on authorship, contributions, and financial \& other disclosures was provided by the authors and is available with the online version of this article at wWw. haematologica.org.

\section{References}

1. Gisslinger H, Gotic M, Holowiecki J, et al. Anagrelide compared with hydroxyurea in WHO-classified essential thrombocythemia: the ANAHYDRET Study, a randomized controlled trial. Blood. 2013; 121(10):1720-1728.

2. Gillespie E. Anagrelide: a potent and selective inhibitor of platelet cyclic AMP phosphodiesterase enzyme activity. Biochem Pharmacol. 1988;37(14):2866-2868

3. Eric M. Mazur AGR, Patricia A. Sohl, Julie L. Newton, Amirthini Narendran. Analysis of the Mechanism of Anagrelide-Induced Thrombocytopenia in Humans. Blood. 1992;79(8):1931-1937.

4. Solberg LA Jr TA, Oles KJ, Tarach JS, Petitt RM, Forstrom LA, Silverstein MN. The effects of anagrelide on human megakaryocytopoiesis. Br J Haematol. 1997;99(1):174-180.

5. Tomer A. Effects of anagrelide on in vivo megakaryocyte proliferation and maturation in essential thrombocythemia. Blood. 2002; 99(5):1602-1609.

6. Takayama N, Nishimura S, Nakamura S, et al. Transient activation of c-MYC expression is critical for efficient platelet generation from human induced pluripotent stem cells. J Exp Med. 2010; 207(13):2817-2830.

7. Nakamura S, Takayama N, Hirata S, et al. Expandable megakaryocyte cell lines enable clinically applicable generation of platelets from human induced pluripotent stem cells. Cell Stem Cell. 2014; 14(4):535-548.

8. Ito Y, Nakamura S, Sugimoto N, et al. Turbulence Activates Platelet Biogenesis to Enable Clinical Scale Ex Vivo Production. Cell. 2018; 174(3):636-648

9. Hirata S, Murata T, Suzuki D, et al. Selective Inhibition of ADAM17 efficiently mediates glycoprotein Ib $\alpha$ retention during ex vivo generation of human induced pluripotent stem cell-derived platelets. Stem Cells Transl Med. 2017;6(3):720-730.

10. Butcher L, Ahluwalia M, Ord T, et al. Evidence for a role of TRIB3 in the regulation of megakaryocytopoiesis. Sci Rep. 2017;7(1):6684.

11. Eisman R, Surrey S, Ramachandran B, Schwartz E, Poncz M Structural and Functional Comparison of the Genes for Human Platelet Factor 4 and PF4alt. Blood. 1990;76(2):336-344.

12. Apostolidis PA, Lindsey S, Miller WM, Papoutsakis ET. Proposed megakaryocytic regulon of p53: the genes engaged to control cell cycle and apoptosis during megakaryocytic differentiation. Physiol Genomics. 2012;44(12):638-650.

13. Apostolidis PA, Woulfe DS, Chavez M, Miller WM, Papoutsakis ET. Role of tumor suppressor p53 in megakaryopoiesis and platelet function. Exp Hematol. 2012;40(2):131-142.

14. Zou X, Qu M, Fang F, et al. Small Molecule Supplements Improve Cultured Megakaryocyte Polyploidization by Modulating Multiple Cell Cycle Regulators. Biomed Res Int. 2017;2017:2320519.

15. Ahluwalia M, Butcher L, Donovan H, Killick-Cole C, Jones PM, Erusalimsky JD. The gene expression signature of anagrelide provides an insight into its mechanism of action and uncovers new regulators of megakaryopoiesis. J Thromb Haemost. 2015;13(6):11031112 . 